\title{
Immunocytochemical localization of tyrosine hydroxylase in the visual cortex of the microbat, Rhinolophus ferrumequinum
}

\author{
Myung-Jun Lee, Kyung-Min Kwon, Won-Tae Lee, Gil-Hyun Kim, Chang-Jin Jeon* \\ Department of Biology, School of Life Sciences, BK21 Plus KNU Creative BioResearch Group, \\ College of Natural Sciences, and Brain Science and Engineering Institute, Kyungpook National \\ University, Daegu, 41566, South Korea
}

\begin{abstract}
Introduction. In order to enhance our understanding of bat vision, we investigated tyrosine hydroxylase (TH)-immunoreactive (IR) fibers in the visual cortex of the microbat.

Material and methods. The study was conducted on 12 freshly-caught adult bats (Rhinolophus ferrumequinum, both sexes, weighing 15-20 g). We used standard immunocytochemistry and confocal microscopy.

Results. TH-IR fibers were distributed throughout all layers of the visual cortex, with the highest density in layer I. Two types of TH-IR fibers were observed: small and large varicose fibers. TH-IR cells were not found in the microbat visual cortex. The microbat substantia nigra and ventral tegmental areas, previously identified sources of TH-IR fibers in the mammalian visual cortex, all contained strongly labeled TH-IR cells. The average diameters of TH-IR cells in the substantia nigra and the ventral tegmental areas were $14.39 \pm 0.13 \mu \mathrm{m}$ (mean \pm SEM) and $11.85 \pm 0.13 \mu \mathrm{m}$, respectively.

Conclusions. Our results suggest that the microbat has a well-constructed neurochemical organization of THIR fibers. This observation should provide fundamental insights into a better understanding of the nocturnal, echolocating bat visual system. (Folia Histochemica et Cytobiologica 2020, Vol. 58, No. 2, 61-72)
\end{abstract}

Key words: visual cortex; tyrosine hydroxylase; microbat; immunocytochemistry

\section{Introduction}

Bats are the only true flying mammals in the order Chiroptera and they help to maintain the healthy ecosystem with their pollination activities [1-4]. There are about 1,000 species of bats which are generally divided into two suborders: Megachiroptera (megabats, approximately 200 species), and Microchiroptera (microbats, approximately 800 species). Megabats, also called fruit bats, have large eyes with visual ability, while microbats have been known to have degenerated

Correspondence address:

Prof. Chang-Jin Jeon, Neuroscience Lab.,

Department of Biology, College of Natural Sciences,

Kyungpook National University, 80, Daehak-ro,

Daegu, 41566, South Korea

phone: +82-53-950-5343, fax: +82-53-953-3066,

e-mail: cjjeon@knu.ac.kr eyes with poor eyesight. Microbats primarily rely on sophisticated laryngeal echolocation, making high-frequency noises and listening to the returning echoes for navigation or prey detection [5-9].

It has been recognized, however, that many microbats also depend on vision. Thus, many functional elements of the retina have been found in the microbat including cone $[10,11]$ and rod photoreceptors [12], ultraviolet-sensitive cone photoreceptors [13,14], and the functional opsin genes $[15,16]$, suggesting the possibility for photopic and scotopic vision. A recent study also described the distribution of the third class of mammalian photoreceptors, intrinsically photosensitive retinal ganglion cells (ipRGCs), in the microbat retina. The microbat has a much higher density of ipRGCs than diurnal animals, demonstrating the high species diversity between nocturnal and diurnal animals [17]. The AII [11, 12] and starburst [18] amacrine cells, which are the most well-understood amacrine 
cells in many other mammalian species, have also been found in the microbat retina. Bipolar cells [12, 19] and ganglion cells [20] have also been identified in the microbat. In addition to the retina, various neuronal cells and fibers containing neurochemical components such as acetylcholine, nitric oxide, and calcium-binding proteins have been localized to the visual system of the microbat visual cortex [21-23] and in the superior colliculus [24], indicating the potential for visual perception and visuomotor integration.

Dopamine (DA), an important neurotransmitter in the nervous system, plays a widespread role in attention, arousal, learning, motivation, motor control, reinforcement, and reward [25-30]. Dopaminergic fibers and cells have been commonly found in the visual cortex of various mammals including rats [31-33], rabbits [34], whales [35], monkeys [31, 36], and humans [37]. Layer I contains the highest density of dopaminergic fibers $[31,35]$. The visual cortex is known to receive inputs from dopaminergic neurons in the midbrain. The substantia nigra and ventral tegmental areas are also sources of dopaminergic fibers in the visual cortex [26, 27, 30, 38-40].

The greater horseshoe bat, Rhinolophus ferrumequinum, is an insectivorous microbat that mainly lives in caves. This species has a wide range in the Palearctic ecozone, occurring from North Africa and Southern Europe through South-west Asia, the Caucasus, Iran, Afghanistan, Pakistan, and the Himalayas, and all the way to South-eastern China, Korea, and Japan [41]. Our laboratory previously described the organization of the dopaminergic system in the microbat central visual system, and we have identified at least four types of TH-IR cells in the retina [18]. Thus, although echolocation is the primary method of navigation for microbat flight, it is also of interest to explore the functional organization of the central visual system to find possible implications for the role of vision in flight navigation [11, 17, 18, 23, 24, 42-44]. In the superior colliculus, TH-IR immunoreactivity was found within the fibers but not within the cells [24]. However, the distribution and morphology of dopaminergic fibers in the microbat visual cortex has not yet been investigated. As part of a larger effort to localize dopaminergic cells and fibers in the entire bat visual system, in this study we aimed to investigate the organization of dopaminergic systems in the microbat visual cortex and to compare the organization of the microbat visual cortex with that of other mammals. Our results provide some basic information about the neurochemical organization of TH-IRs immunoreactivities in relation to the functional role of visual ability in the nocturnal echolocating bat.

\section{Materials and methods}

Animal and tissue preparation. For this study, a total of 12 freshly-caught adult bats (Rhinolophus ferrumequinum, both sexes, weighing 15-20 g) were examined. All of the bats were captured in a cave in the district of Gimcheon, South Korea. After 5-6 h to transport and stabilize the bats, they were anesthetized with a mixture of ketamine hydrochloride (30-40 mg/kg; Yuhan, Seoul, Korea) and xylazine (3-6 mg/kg; Bayer, Leverkusen, Germany) prior to perfusion. The bats were then perfused intracardially with $4 \%$ paraformaldehyde (Sigma-Aldrich, St. Louis, MO, USA) and 0.3-0.5\% glutaraldehyde (Sigma-Aldrich) in $0.1 \mathrm{M}$ sodium phosphate buffer ( $\mathrm{pH} 7.4$ ) containing $0.002 \%$ calcium chloride (Yakuri Pure Chemicals, Kyoto, Japan). Following a pre-rinse with approximately $30 \mathrm{ml}$ of phosphate-buffered saline (PBS, $\mathrm{pH} 7.4$ ) over a period of 3-5 min, each bat was perfused with $30-50 \mathrm{ml}$ of fixative for 20-30 min via a syringe needle inserted through the left ventricle and aorta. The animal was decapitated and the head was placed in fixative for 2-3 h. The brain was then removed from the skull, stored for 2-3 h in the same fixative, and then left overnight in $0.1 \mathrm{M}$ phosphate buffer ( $\mathrm{pH} 7.4$ ) containing $8 \%$ sucrose and $0.002 \%$ calcium chloride. The brain was then mounted onto a chuck and cut into $50 \mu \mathrm{m}$ thick coronal sections using a Vibratome 3000 Plus Sectioning System (Vibratome, St. Louis, MO, USA). All animal experiments were approved by the Animal Care and Use Committee of Kyungpook National University (permission No. 2016-0151). The "Principles of laboratory animal care" (NIH publication No. 85-23, revised 1985) were followed throughout the research protocol period.

Horseradish peroxidase staining. The primary antibody used in this study was rabbit anti-tyrosine hydroxylase (TH; Pel-Freez, Rogers, AR, USA) [18, 24, 45]. Tissues were processed free floating in small vials at room temperature with gentle agitation. For immunocytochemistry, tissues were incubated in $1 \%$ sodium borohydride $\left(\mathrm{NaBH}_{4}\right.$; Sigma-Aldrich) for $30 \mathrm{~min}$. Afterwards, the tissues were rinsed for $3 \times 10 \mathrm{~min}$ in PBS, then incubated for $2 \mathrm{~h}$ in PBS with $4 \%$ normal goat serum (Vector Laboratories, Burlingame, CA, USA) with $0.5 \%$ Triton X-100 (Yakuri Pure Chemicals) added. Tissues were next incubated for $24 \mathrm{~h}$ in the primary antiserum (diluted 1:200) in PBS with 4\% normal serum with $0.5 \%$ Triton X-100 added. Following three $10 \mathrm{~min}$ rinses in PBS, the tissues were incubated in a 1:200 dilution of biotinylated secondary IgG in a blocking solution. The secondary antibody used was biotinylated goat anti-rabbit (Vector Laboratories). The tissues were then rinsed three times for 10 min each in PBS and incubated for $2 \mathrm{~h}$ in a 1:50 dilution of avidin-biotinylated horseradish peroxidase complex (Vector Laboratories) in PBS. The tissues were 
then rinsed in $0.25 \mathrm{M}$ Tris buffer for $3 \times 10 \mathrm{~min}$. Finally, staining was visualized by reaction with 1,3 '-diaminobenzidine tetrahydrochloride (DAB) and hydrogen peroxide in $0.25 \mathrm{M}$ Tris buffer for 3-10 min using a DAB reagent set (Kirkegaard \& Perry, Gaithersburg, MD, USA). All tissues were then rinsed in $0.25 \mathrm{M}$ Tris buffer before mounting. As a negative control, some sections were incubated in the same solution without the addition of the primary antibody, and these control tissues showed no TH-IR immunoreactivity. Negative control and preabsorption tests for the specificity of this antibody conducted in the microbat central nervous system have been described in previous reports [24, 46]. Following the immunocytochemical procedures, the tissues were mounted on Superfrost Plus slides (Fisher, Pittsburgh, PA, USA) and dried overnight in a $37^{\circ} \mathrm{C}$ oven. The mounted sections were dehydrated, cleared, and coverslipped with Permount (Fisher).

Quantitative analysis. TH-IR fibers in the bat visual cortex were examined and photographed with a Zeiss Axioplan microscope (Carl Zeiss Meditec, Inc., Dublin, CA, USA). Among the 12 bats, we chose three animals and analyzed the quantitative data from nine best-labeled tissue sections (three tissue sections from each of three animals). Conventional or differential interference contrast (DIC) optic images were obtained using Zeiss AxioCam HRc digital camera with Zeiss Plan-Apochromat objectives and Zeiss AxioVision 4 software (Carl Zeiss Meditec, Inc.).

To quantify the density of IR fibers, we sampled sequential $210 \times 210 \mu \mathrm{m}$ fields and analyzed from nine sections in three bats (three tissue sections from each of three animals) across the superficial and deeper layers of the visual cortex. Line drawings showing the IR fibers were generated to clearly distinguish the distribution of the TH-IR fibers in the representative bat visual cortical section using Adobe Photoshop CS software (Adobe Systems Inc., San Jose, CA, USA).

In three animals, the morphological types and the average diameter and area of TH-IR neurons were analyzed. The morphological types were determined for 273 (SN) and 316 (VTA) neurons analyzed from nine sections in three bats and the average diameter and area of TH-IR neurons were determined for 210 (SN) and 236 (VTA) neurons analyzed from nine sections in three bats (three tissue sections from each of three animals). All analyses were performed using a $40 \times$ Zeiss Plan-Apochromat objective. To obtain the best images, we analyzed the cells under DIC optics. Only cell profiles containing a nucleus and at least one faintly visible nucleolus were included in the analysis. Since our goal was to obtain an estimate of each morphological cell type, no attempt was made to assess the total number of cells in each neuronal subpopulation. The average diameter and area of labeled neurons were computed using a digital camera (Carl Zeiss Meditec, Inc.). A cursor was moved manually around the outer contour of each cell using Zeiss AxioVison system. Images were adjusted with respect to brightness and contrast using Adobe Photoshop CS software (Adobe Systems Inc.).

\section{Results}

\section{The distribution of TH-IR fibers in the microbat visual cortex}

The laminar structure of the microbat visual cortex and the TH-IR immunoreactivity are shown in Figures 1A and $1 \mathrm{~B}$, respectively. These images demonstrate that the microbat visual cortex has well-developed dopaminergic fibers. TH-IR fibers were distributed throughout all layers of the microbat visual cortex. Figures $1 \mathrm{C}$ and 1D show highly magnified sections of the square regions in Figure $1 \mathrm{~B}$. Figure $1 \mathrm{~B}$ is a dark-field photomicrograph, captured using $20 \times$ objective. Figure $1 \mathrm{C}$, including the TH-IR fibers located within layers I-III, and Figure 1D, showing the TH-IR fibers located within layers IV-VI, are bright-field photomicrographs, captured using a $40 \times$ objective. A representation of the density of the TH-IR fibers is shown in Figure 1E. The dopaminergic fibers in the microbat visual cortex form a plexus of labeled fibers with differential densities in the different layers. Quantitative maps of fiber distribution indicate the density of the TH-IR fibers in each layer (Fig. 2). Figure 2 shows the laminar distribution pattern of the TH-IR fibers in the microbat visual cortex, which were analyzed using an image processing program developed at the National Institutes of Health. Three different sections each from three individual animals (total 9 sections, each represented by a different color in Fig. 2B) were analyzed in this study. Figure $2 \mathrm{C}$ shows a histogram of the density of the TH-IR fibers in the microbat visual cortex. The frequency of labeled fibers varies in each layer: $20.78 \pm 0.70 \%$ (mean \pm SEM) of the labeled fibers appear in layer I, $13.65 \pm 0.42 \%$ in layer II, $15.89 \pm 0.74 \%$ in layer III, $16.14 \pm 0.63 \%$ in layer IV, $16.45 \pm 0.66 \%$ in layer $\mathrm{V}$, and $17.07 \pm$ $\pm 0.74 \%$ in layer VI. Layer I had the highest density of TH-IR fibers, while layer II had the lowest TH-IR fiber density (Figs. 2B, 2C).

\section{The morphology of TH-IR fibers in the microbat visual cortex}

We identified at least two types of TH-IR varicose fibers in the microbat visual cortex: small varicose fibers (arrowheads in Figs. 3B, 3C and 3D) and large varicose fibers (arrows in Figs. 3A, 3B, 3D and 3E). Varicose fibers were distributed throughout the rostrocaudal section of the microbat visual cortex and numerous TH-IR varicosities were distributed irregularly along the fibers. The TH-IR fibers in the 


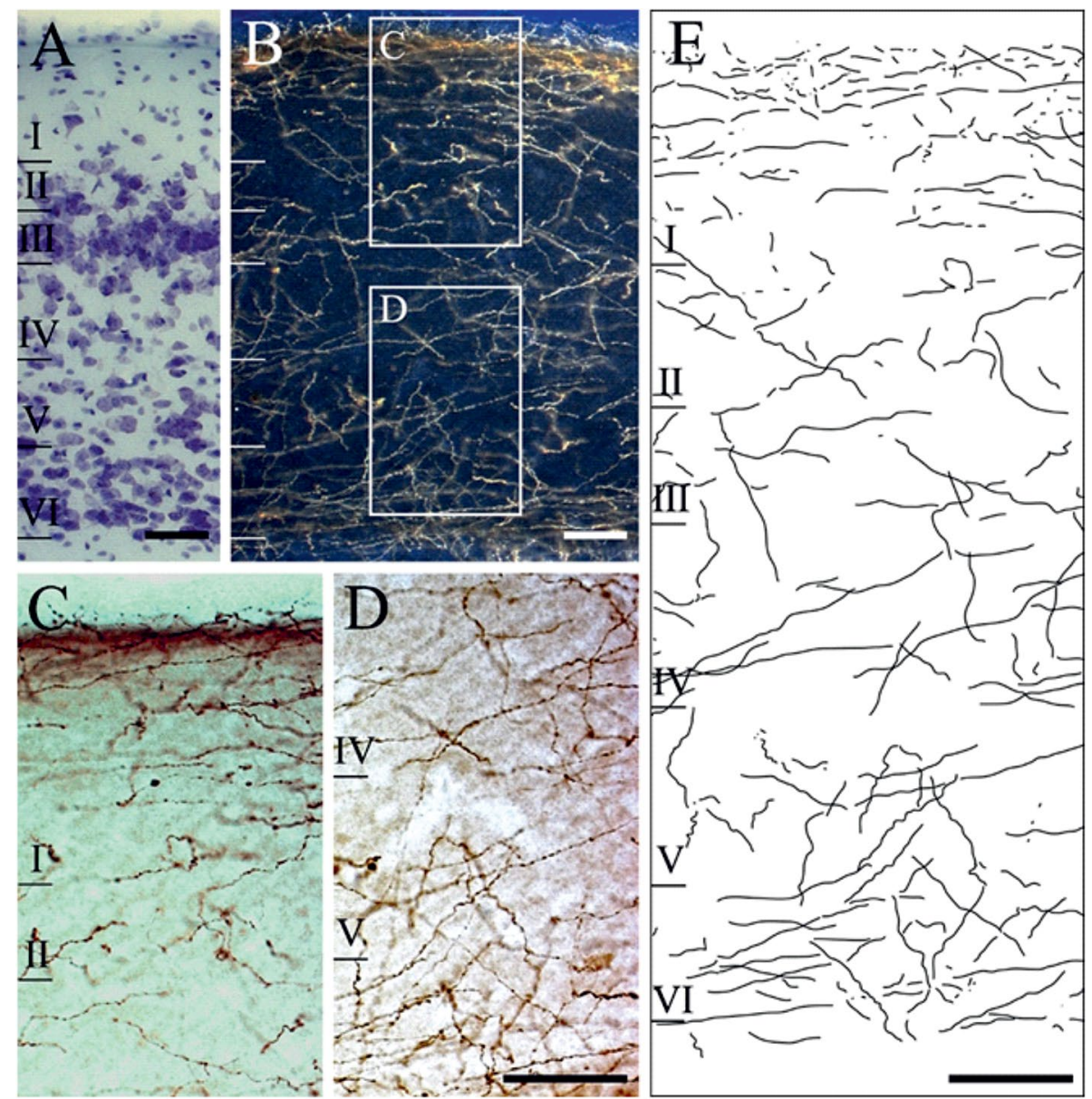

Figure 1. Distribution of TH-IR fibers in the microbat visual cortex. A. The approximate limits of the layers are indicated in the thionin-stained section. B. Low-magnification dark-field photomicrograph of TH-IR fibers in the microbat visual cortex. C, D. Medium magnification bright-field photomicrograph of TH-IR fibers showing the areas marked in (B). Varicose dopaminergic fibers generally observed in the microbat visual cortex. E. Drawing of the TH-IR fibers in the microbat visual cortex. Bar $=50 \mu \mathrm{m}$.
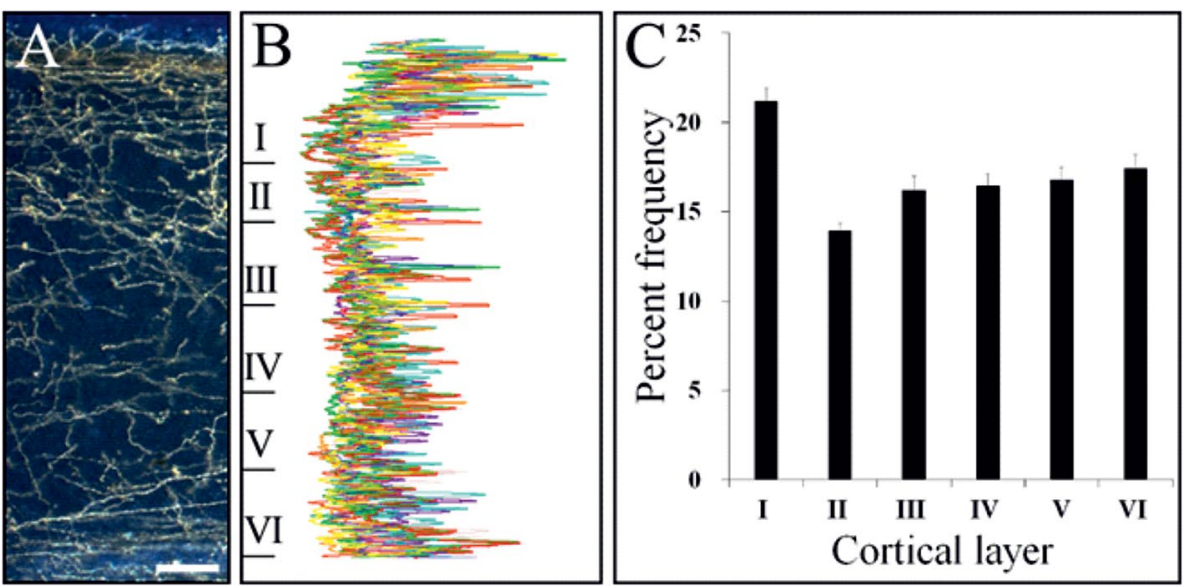

Figure 2. Intensity profile of TH-IR fibers in the visual cortex of the microbat. A plot of the intensity of the TH-IR fibers in different coronal sections of the microbat visual cortex, characterized using the image processing program Image $\mathbf{J}$ (NIH, Bethesda, MD, USA). A. Low-magnification dark-field photomicrograph of the microbat visual cortex shows well-labeled TH-IR fibers. B. Results of intensity graph analyses from nine coronal sections of the microbat visual cortex are shown. C. Density histogram of TH-IR fibers in the microbat visual cortex. Highest density was found in layer I. Bar $=50 \mu \mathrm{m}$. 

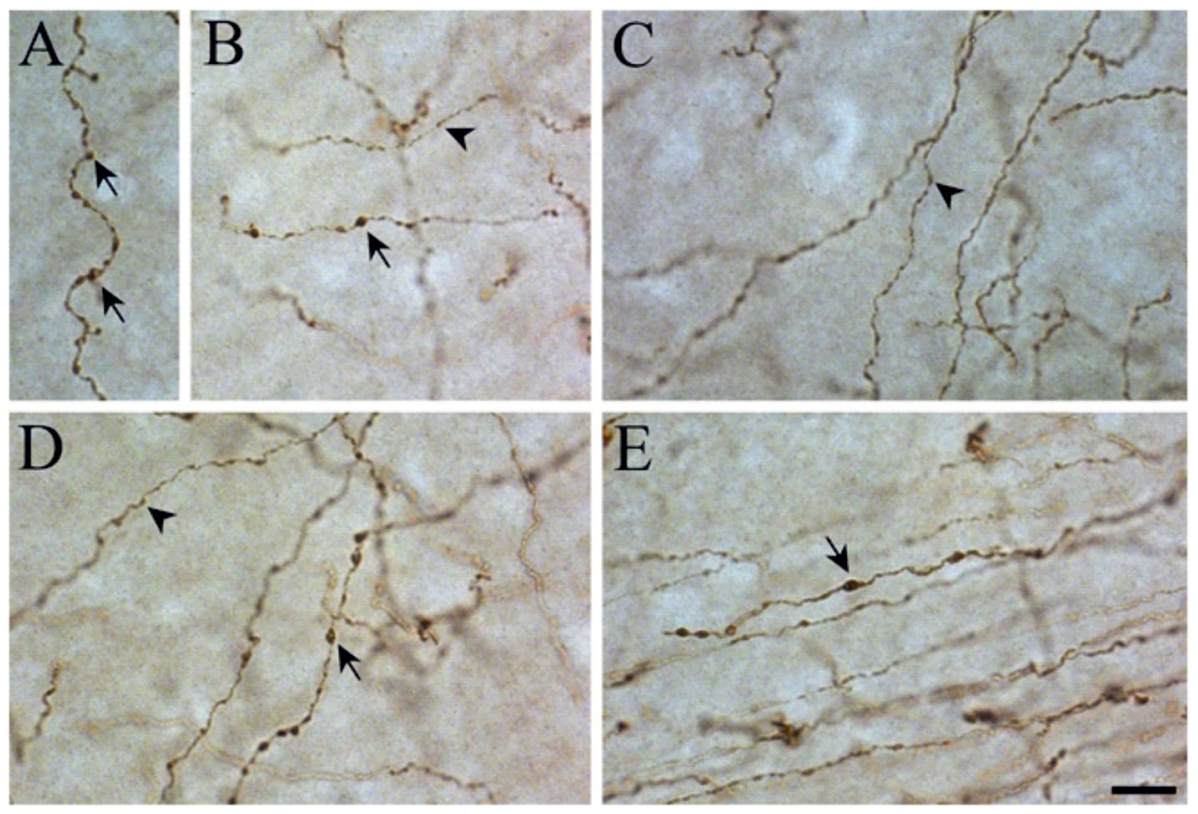

Figure 3. High-power differential interference contrast (DIC) photomicrographs of TH-IR fibers in the microbat visual cortex. A, E. Arrows indicate large varicosities in the microbat visual cortex. C. Arrowheads indicate small varicosities in the microbat visual cortex. B, D. TH-IR fibers with small (arrowheads) and large (arrows) varicosities in the microbat visual cortex. Bar $=10 \mu \mathrm{m}$.

microbat visual cortex exhibited various orientations, including vertical, horizontal, and oblique orientations. The microbat visual cortex does not appear to have dopaminergic neurons; no labeled neurons were found in this study.

\section{Source of dopaminergic inputs to the microbat visual cortex}

There are several subdivisions of dopaminergic neuron groups. The main groups of dopaminergic neurons are located in the following areas in the brain: A8 (retrorubral), A9 (substantia nigra), A10 (ventral tegmental area), A11 (caudal diencephalic group), A12 (tuberal cell group), A13 (zona incerta), A14 (hypothalamic nuclei), A15 (anterior hypothalamic group), and A16 (olfactory bulb) [46-48]. Among these, the substantia nigra and ventral tegmental areas are known to project dopaminergic fibers into the visual cortex [26, 27, 30, 38-40].

\section{Substantia nigra}

TH-IR neurons were distributed in the substantia nigra of the bat brain. Figure 4A represents an immunostained section containing the substantia nigra. Figure $4 \mathrm{~B}$, which is the magnified image of the area marked by a square in Figure 4A, shows intense labeling in various types of neurons in the substantia nigra. The large majority of the TH-IR neurons were round or oval cells, although other cell types were also observed, including stellate, horizontal fusiform, and vertical fusiform. Figure 5A shows representative multipolar round or oval cells. Figure 5B shows a small-sized stellate neuron. The stellate cells had polygon-shaped cell bodies with many dendrites coursing in all directions. Figure 5C shows a pyriform neuron with a pear-shaped cell body and a thick proximal dendrite directed toward the pial surface. The ascending process has many small branches, forming a dendritic bouquet. Figure 5D shows a vertical fusiform cell that displays a vertical fusiform cell body with a main, long process ascending towards the pial surface and a long descending process. Figure 5E shows a horizontal cell that displays a horizontally oriented small, fusiform cell body and horizontally oriented processes.

Quantitatively, $52.01 \pm 3.06 \%$ ( 142 of 273 cells) of TH-IR neurons were round or oval, $15.82 \pm 1.06 \%$ (51 of 273 cells) were vertical fusiform, $16.12 \pm 0.74 \%$ (44 of 273 cells) were stellate, $11.72 \pm 1.16 \%$ (32 of 273 cells) were horizontal cells, and $1.47 \pm 0.12 \%$ (4 of 273 cells) were pyriform cells (Fig. 7). The average diameter of the $210 \mathrm{TH}$-IR neurons in the substantia nigra ranged from 8.41 to $20.13 \mu \mathrm{m}$, with a mean diameter of $14.39 \pm 0.13 \mu \mathrm{m}$. The area of these cells ranged from 55.51 to $318.26 \mu \mathrm{m}^{2}$, with a mean area of $165.90 \pm 3.23 \mu \mathrm{m}^{2}$ (Fig. 8).

\section{Ventral tegmental area}

Figure 4C presents an immunostained section containing the ventral tegmental area. Figure $4 \mathrm{D}$ is the magnified image of the area marked by square in Figure 4C 

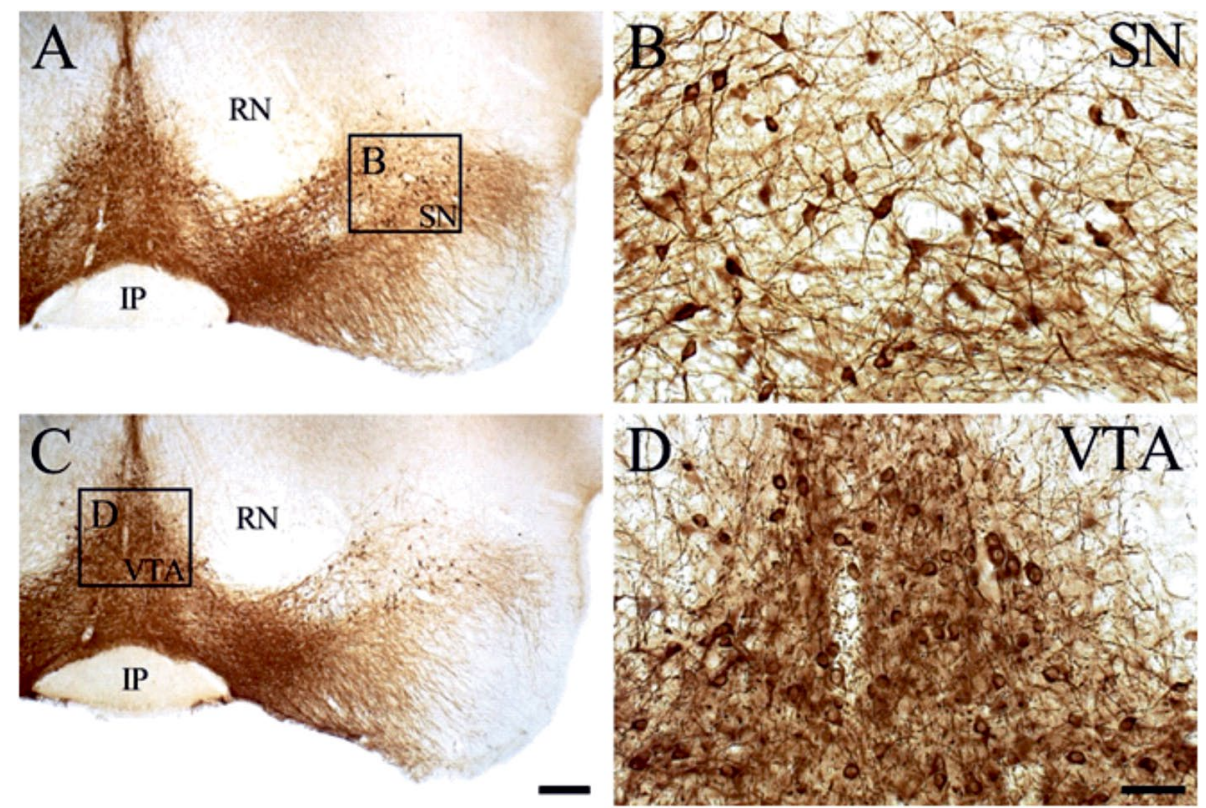

Figure 4. Photomicrographs show presumed sources of TH-IR fibers in the microbat visual cortex. A. Low-magnification photomicrograph of coronal sections showing the substantia nigra. B. High-magnification photomicrograph of the image in the square marked in (A). C. Low magnification photomicrograph of a coronal section showing the ventral tegmental area. D. High magnification photomicrograph of the image in the square marked in (C). RN — red nucleus; SN - substantia nigra; VTA - ventral tegmental area. Bars: $\mathrm{C}=200 \mu \mathrm{m}, \mathrm{D}=50 \mu \mathrm{m}$.

and shows intense labeling in various types of neurons in the ventral tegmental area. The majority of TH-IR neurons in the microbat ventral tegmental area were round or oval cells. Figure 6A shows several representative round or oval cells, and Figure 6B shows a large-sized stellate neuron with a polygon-shaped cell body and numerous dendrites coursing in all directions. Figure $6 \mathrm{C}$ shows a vertical fusiform cell that displays a vertical fusiform cell body with a main, long process ascending towards the pial surface and a long descending process. Figure 6D shows a horizontal cell that displays a horizontally oriented fusiform cell body and horizontally oriented processes. The pyriform cells (Fig. 6E) have pear-shaped cell bodies with a thick, proximal dendritic stump directed superficially towards the pial surface. Pyriform and horizontal cell types, however, were rare.

Quantitatively, $60.76 \pm 3.47 \%$ (192 of 316 cells) of the TH-IR neurons were round or oval, 15.82 \pm $0.82 \%$ ( 50 of 316 cells) were vertical fusiform, $8.23 \pm$ $0.11 \%$ ( 26 of 316 cells) were stellate, $13.61 \pm 1.55 \%$ (43 of 316 cells) were horizontal cells, and $1.58 \pm$ $0.11 \%$ ( 5 of 316 cells) were pyriform cells (Fig. 7). The average diameter of the 236 TH-IR neurons in the ventral tegmental area ranged from 8.18 to 17.55 $\mu \mathrm{m}$, with a mean diameter of $11.85 \pm 0.13 \mu \mathrm{m}$. The area of these cells ranged from 52.61 to $242.04 \mu \mathrm{m}^{2}$, with a mean area of $113.62 \pm 2.54 \mu \mathrm{m}^{2}$ (Fig. 8).

\section{Discussion}

This study demonstrates that TH-IR fibers are distributed in the microbat visual cortex. We identified at least two types of TH-IR varicose fibers, and the TH-IR cells identified in the substantia nigra and ventral tegmental area are thought to be the sources of these TH-IR fibers.

The purpose of this study was to investigate if TH-IR fibers are distributed in the microbat visual cortex as previously observed in the visual cortex of other animals [31-37]. The results show that TH-IR fibers with varicosities are present throughout all layers of the microbat visual cortex. A dopaminergic fiber density gradient was observed in the microbat visual cortex: layer I had the most densely innervated lamina, while layer II was the least innervated lamina. Dopaminergic fibers have been found in the visual cortex of many other mammals including rats [31-33], rabbits [34], whales [35], monkeys [31, 36], and humans [37]. In the rat visual cortex, dopaminergic fibers were located in layers I-III and in layer VI [31]. In the rabbit visual cortex, layers I-II and layer IV are the most heavily innervated [34]. In the whale visual cortex, most of the dopaminergic fibers were primarily located in layer I [35], and a similar pattern was found in the monkey visual cortex [31]. Thus, 

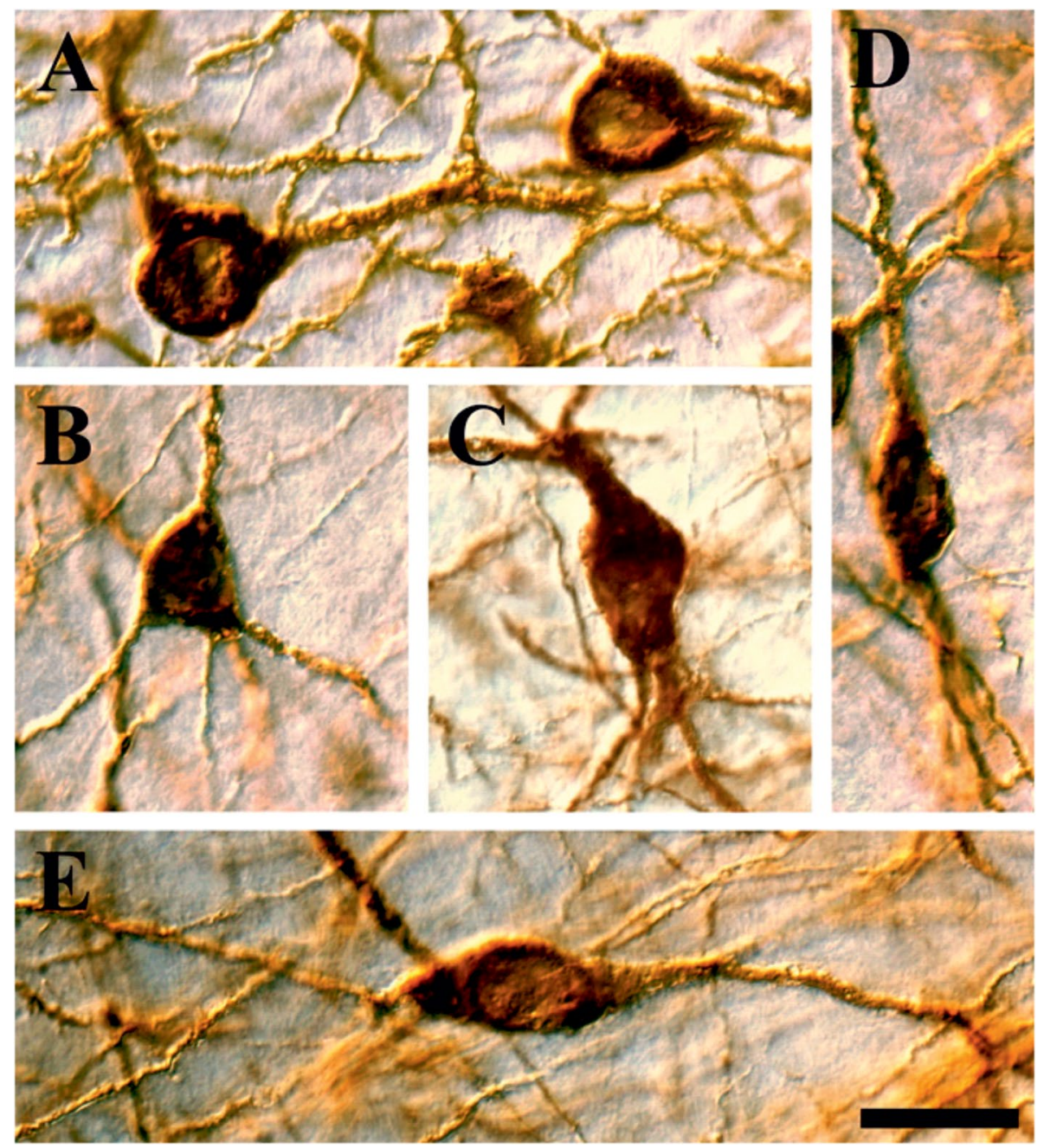

Figure 5. High-power differential interference contrast (DIC) photomicrographs of TH-IR cell types in the microbat substantia nigra. A. Round cells. At least three processes in various directions are seen (right side). The majority of TH-IR neurons were round or oval cells with many dendrites coursing in all directions. B. A multipolar stellate cell. Stellate neurons had polygon-shaped cell bodies with many dendrites coursing in all directions. C. A pyriform cell with a thick primary dendrite oriented toward the pial surface. This ascending process has many small branches, forming a dendritic bouquet. D. A vertical fusiform cell with a vertical fusiform cell body and vertically oriented processes. E. A horizontal fusiform cell with a horizontal fusiform cell body and horizontally oriented processes. Bar $=20 \mu \mathrm{m}$.

although the pattern of dopaminergic innervation has not been studied extensively across diverse animal species, the distribution pattern of the TH-IR fibers in the microbat visual cortex is similar to that of most of the mammals studied to date.

We found TH-IR varicosities throughout the microbat visual cortex, and identified at least two different types: small and large varicose TH-IR fibers. Varicose dopaminergic fibers are common in several brain regions in mammals [24, 31, 32, 35], including the visual cortex of rats [31,32], whales [35], and monkeys [36]. In our previous study on the distribution of TH in microbats, we also identified two types of varicose dopaminergic fibers in the superior colliculus
[24]. As differences in shape, diameter, and synaptic microstructure of varicosities are thought to affect synaptic profiles [49], we propose that the dopaminergic output may be influenced by the morphology of the varicose dopaminergic fibers. However, electron microscopy studies will be required to confirm the synaptic profiles of the TH-IR fibers in the microbat visual cortex.

We did not find TH-IR neurons in the microbat visual cortex, and our results are similar to those of previously studies of mammals such as monkeys [35] and rabbits [34]. However, the TH-IR neurons were found in rat, whale, and human visual cortex [32,37]. In rat visual cortex, the TH-IR neurons are located 


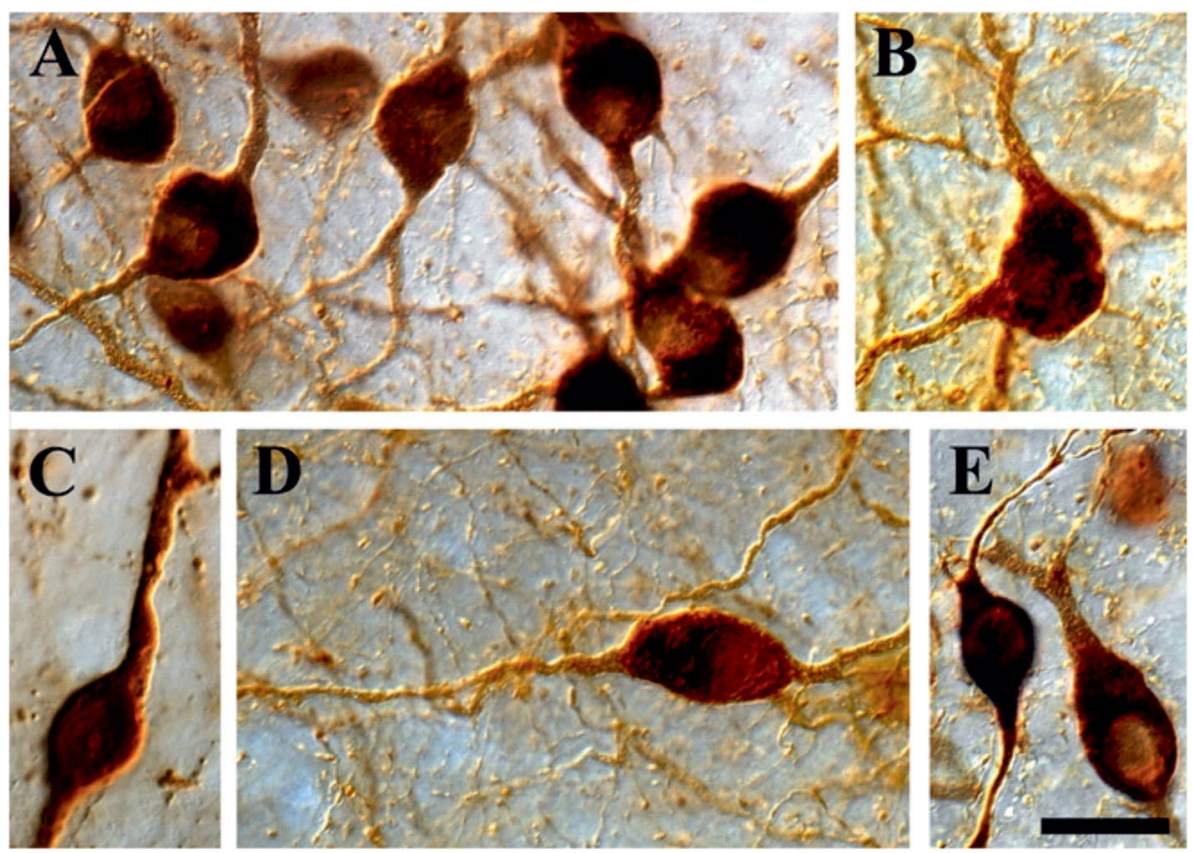

Figure 6. High-power differential interference contrast (DIC) photomicrographs of TH-IR cell types in the microbat ventral tegmental area. A. Several round or oval cells can be identified in this Figure. The majority of TH-IR cells were round or oval cells. B. A multipolar stellate cell. Stellate neurons had polygon-shaped cell bodies with many dendrites coursing in all directions. C. A vertical fusiform cell with a vertical fusiform cell body and vertically oriented processes. D. A horizontal fusiform cell with a horizontal fusiform cell body and horizontally oriented processes. E. Pyriform cells with a thick primary dendrite oriented toward the pial surface. This ascending process had small branches, forming a dendritic bouquet. Bar $=20 \mu \mathrm{m}$.

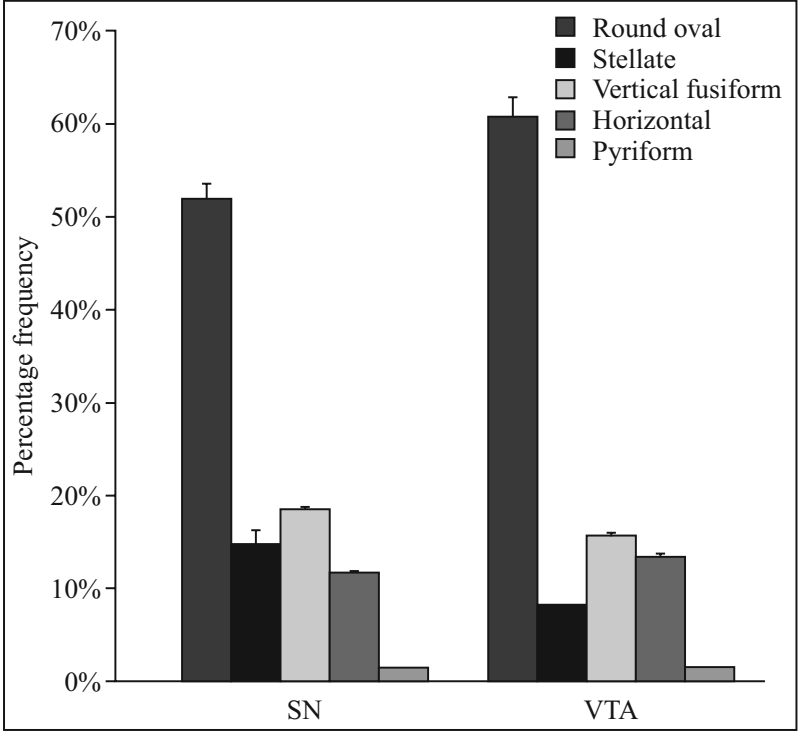

Figure 7. Histogram of the distribution of TH-IR neurons in the microbat substantia nigra and ventral tegmental area. In both areas, round or oval neurons presented the highest concentration of TH-IR cells, whereas the pyriform neurons comprised the smallest proportion. SN — substantia nigra; VTA - ventral tegmental area. in layers I-VI. Most of these TH-IR neurons have bipolar or multipolar morphology and either fusiform or oval-shaped cell bodies [32]. In the whale visual cortex, TH-IR neurons are mainly observed in layer I [35]. TH-IR neurons in the visual cortex are mainly considered to be interneurons, and have been found to coexpress GABA or its biosynthetic enzyme glutamic acid decarboxylase (GAD) [50]. The functional significance of these inter-species differences in the presence of dopaminergic neurons in the visual cortex has not yet been understood. However, these patterns may reflect variations in the connections and synaptic loops of the dopaminergic system in the animal visual cortex. Another study demonstrated that dopamine modulates GABA release from interneurons via the D2-like dopamine receptors in the prefrontal cortex [51]. Thus, dopaminergic fibers may synapse with GABAergic interneurons and modulate GABA levels in the microbat visual cortex.

In the midbrain, TH-IR neurons found in the substantia nigra and ventral tegmental area form several dopaminergic pathways, such as the nigrostriatal, mesolimbic, and mesocortical pathways, that are 

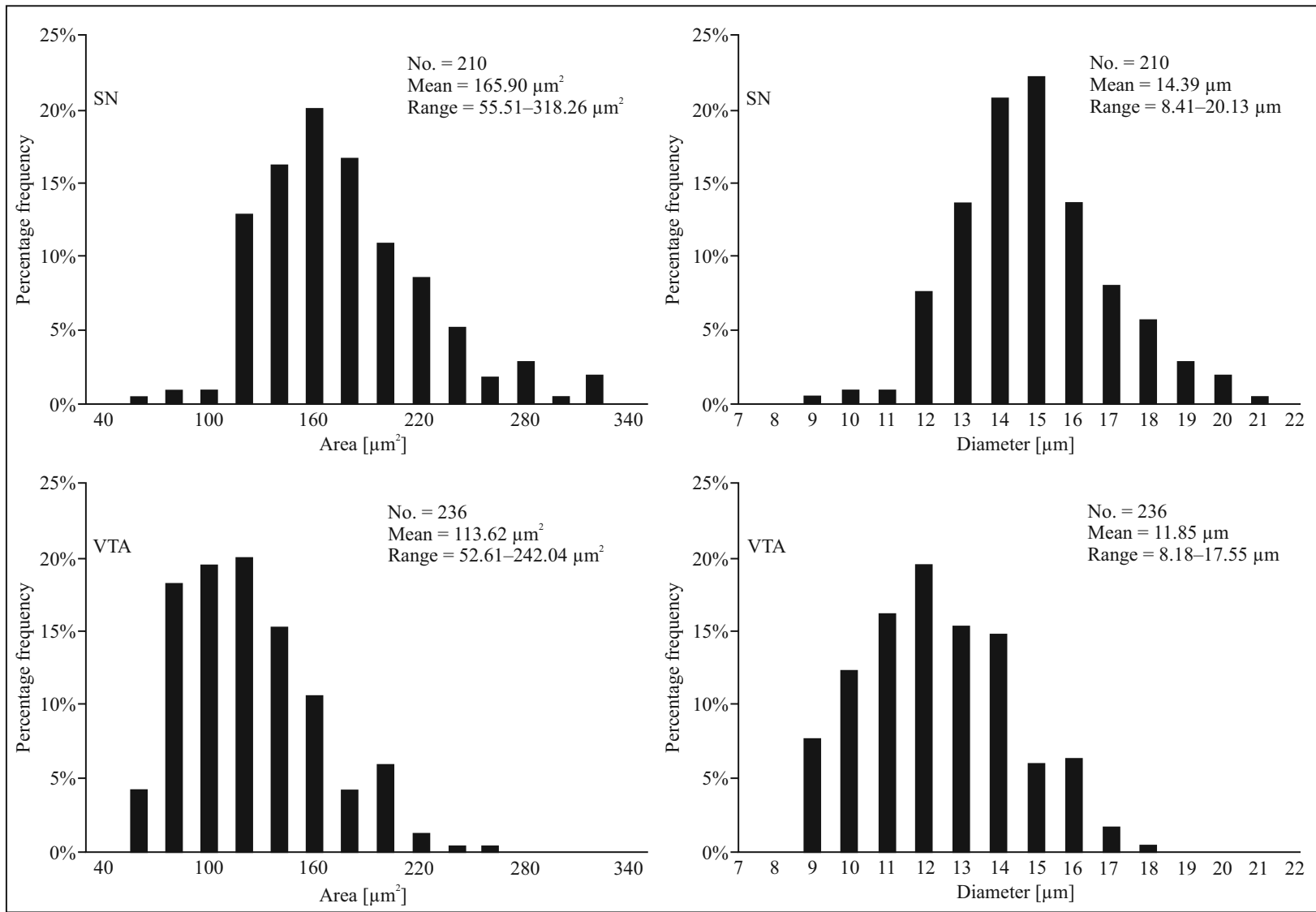

Figure 8. Histogram of TH-IR cell area and diameter in the microbat brain. The diameter of 210 TH-IR neurons in the substantia nigra ranged from 8.41 to $20.13 \mu \mathrm{m}$, with a mean of $14.39 \pm 2.01 \mu \mathrm{m}$ (mean $\pm \mathrm{SD}$ ). The area of these cells ranged from 55.51 to $318.26 \mu \mathrm{m}^{2}$, with a mean of $165.90 \pm 46.86 \mu \mathrm{m}^{2}$. The diameter of $236 \mathrm{TH}$-IR neurons in the ventral tegmental area ranged from 8.18 to $17.55 \mu \mathrm{m}$, with a mean of $11.85 \pm 2.03 \mu \mathrm{m}$. The area of these cells ranged from 52.61 to $242.04 \mu \mathrm{m}^{2}$, with a mean of $113.62 \pm 39.11 \mu \mathrm{m}^{2}$. The best-stained cells were measured. SN — substantia nigra; VTA — ventral tegmental area.

Table 1. Quantitative data of tyrosine hydroxylase-immunoreactive cells in microbat substantia nigra and ventral tegmental area

\begin{tabular}{|l|c|c|c|c|}
\hline \multirow{4}{*}{ Substantia Nigra } & Animal number & \multirow{2}{*}{ Number of TH cells } & \multicolumn{2}{|c|}{ Diameter \pm SEM } \\
\cline { 3 - 5 } & & & $(\boldsymbol{m})$ & Area \pm SEM $\left(\boldsymbol{\mu} \mathbf{m}^{2}\right)$ \\
\hline \multirow{5}{*}{ Ventral Tegmental Area } & 1 & 72 & $14.76 \pm 0.23$ & $174.41 \pm 5.65$ \\
\cline { 2 - 5 } & 2 & 64 & $14.05 \pm 0.21$ & $157.47 \pm 5.06$ \\
\cline { 2 - 5 } & 3 & 74 & $14.32 \pm 0.25$ & $164.91 \pm 5.81$ \\
\cline { 2 - 5 } & Average & 70 & $14.39 \pm 0.13$ & $165.90 \pm 3.23$ \\
\cline { 2 - 5 } & 1 & 77 & $11.00 \pm 0.18$ & $97.06 \pm 3.27$ \\
\cline { 2 - 5 } & 2 & 78 & $12.45 \pm 0.23$ & $125.18 \pm 4.69$ \\
\cline { 2 - 5 } & 3 & 81 & $12.09 \pm 0.23$ & $118.22 \pm 4.50$ \\
\cline { 2 - 5 } & Average & 78.66 & $11.85 \pm 0.13$ & $113.62 \pm 2.54$ \\
\hline
\end{tabular}

known to project to the visual cortex. These pathways are associated with emotion-based behaviors and the control of voluntary motor movements. In previous studies identifying dopaminergic nuclei in megabats and microbats, TH-IR nuclei were found in the substantia nigra and ventral tegmental areas [24, 46-48, 52]. Morphometric analysis of the TH-IR cells in the substantia nigra showed a medium-large cellular area 
(range 151.26-255.29 $\mu \mathrm{m}^{2}$ ), while the TH-IR cells in the ventral tegmental area had a small-medium cellular area (range 92.70-187.67 $\mu \mathrm{m}^{2}$ ) [52]. These results are similar to those observed in other mammals [53]. Our results also show that the TH-IR cells in the substantia nigra are larger than in the ventral tegmental area. In the present study we identified various cell types including round or oval, stellate, vertical fusiform, and horizontal cells. Although the presence of dopaminergic circuits need to be verified by retrograde trace injection, our current results and pervious findings $[26,27,30,38-40]$ suggest that dopaminergic cells found within the midbrain may be the source of the dopaminergic innervation in the microbat visual cortex. In addition, dopaminergic neurons of different soma sizes that originate in the midbrain are presumed to project varying sizes of dopaminergic fibers into the microbat visual cortex.

Dopaminergic neurons from the substantia nigra target layer I in the rat brain, while neurons originating in the ventral tegmental area and midline nuclei target layers V-VI [31]. Assuming that the central dopaminergic system in the microbat visual cortex is similar to that of the rat, it is possible that the pattern of TH-IR fiber distribution in the bat visual cortex represents region-specific laminar localization of dopaminergic afferents. The high density of THIR fibers in layer I of the bat visual cortex suggests that the bat visual cortex may receive much denser dopaminergic projections from the substantia nigra compared to the ventral tegmental area. However, the functional significance of the higher density of dopaminergic fibers in layer I of the visual cortex is not completely understood.

The functional role of TH-IR fibers in the visual cortex has not been fully elucidated yet. The dopaminergic system in the visual cortex may be involved in predicting reward information $[54,55]$. Dopamine enhances the visual information content of oscillatory activity in the visual cortex [56]. In the bat, the striatal dopamine receptor-mediated pathway is an important factor in the production of bat echolocation pulses [57]. However, physiological studies of the role of dopamine in the visual cortex of bats have not been conducted. Our results demonstrate a well-organized dopaminergic system in the microbat visual cortex, which suggests that dopaminergic fibers in the visual cortex may play important roles such as predicting reward information and promoting the visual sensory information, as previously described in other mammals [54-56]. However, defining how dopamine modulates various brain functions in the microbat visual cortex requires further studies focusing on electrophysiological approaches.
In conclusion, our results show that well-organized TH-IR fibers are present in the microbat visual cortex. TH-IR cells were not observed in the microbat visual cortex. The microbat substantia nigra and ventral tegmental areas, which are known sources of dopaminergic fibers found in the mammalian visual cortex, contained many well-labelled TH-IR neurons. These results combined with previous studies of the microbat visual system may contribute valuable information to the theory that the microbat visual system has functional roles and is capable of visual processing.

\section{Conflicts of Interest}

The authors declare that there are no conflicts of interest.

\section{Acknowledgements}

We thank Essay Review for proofreading the manuscript.

\section{References}

1. Adams RA. Wing ontogeny, shifting niche dimensions, and adaptive landscapes. In: Adams RA, Pedersen SC, ed. Ontogeny, Functional Ecology, and Evolution of Bats. Cambridge: Cambridge University Press. ; 2000: 275-315.

2. Fenton MB, Simmons NB. Bats: A World of Science and Mystery. Chicago: University of Chicago Press; 2015.

3. Nowack RM. Walker's Mammals of the World. 6th ed. Baltimore: The Johns Hopkins University Press; 1999.

4. Simmons NB. Bat hylogeny: an evolutionary context for comarative studies. In Adams RA, Pedersen SC, ed. Ontogeny, Functional Ecology, and Evolution of Bats. Cambridge: Cambridge University Press; 2000: 9-58.

5. Altringham JD. Bats: Biology and Behaviour. New York: Oxford University Press; 1996.

6. Altringham JD. Bats: From Evolution to Conservation. 2nd ed. New York: Oxford University Press; 2011.

7. Jones G, Teeling EC. The evolution of echolocation in bats. Trends Ecol Evol. 2006; 21(3): 149-156, doi: 10.1016/j. tree.2006.01.001, indexed in Pubmed: 16701491.

8. Surlykke A, Boel Pedersen S, Jakobsen L. Echolocating bats emit a highly directional sonar sound beam in the field. Proc Biol Sci. 2009; 276(1658): 853-860, doi: 10.1098/ rspb.2008.1505, indexed in Pubmed: 19129126.

9. Teeling EC. Bats (Chirotera). In Hedges SB, Kumar S, editors. The Time Tree of Life. Oxford University Press, Oxford 2009: 499-503.

10. Feller KD, Lagerholm S, Clubwala R, et al. Characterization of photoreceptor cell types in the little brown bat Myotis lucifugus (Vespertilionidae). Comp Biochem Physiol B Biochem Mol Biol. 2009; 154(4): 412-418, doi: 10.1016/j. cbpb.2009.08.006, indexed in Pubmed: 19720154.

11. Kim TJ, Jeon YK, Lee JY, et al. The photoreceptor populations in the retina of the greater horseshoe bat Rhinolophus ferrumequinum. Mol Cells. 2008; 26(4): 373-379, indexed in Pubmed: 18612246.

12. Müller B, Butz E, Peichl L, et al. The rod pathway of the microbat retina has bistratified rod bipolar cells and tristratified AII amacrine cells. J Neurosci. 2013; 33(3): 1014-1023, doi: 
10.1523/JNEUROSCI.2072-12.2013, indexed in Pubmed: 23325239.

13. Müller B, Glösmann M, Peichl L, et al. Bat eyes have ultraviolet-sensitive cone photoreceptors. PLoS One. 2009; 4(7): e6390, doi: 10.1371/journal.pone.0006390, indexed in Pubmed: 19636375

14. Winter Y, López J, Von Helversen O. Ultraviolet vision in a bat. Nature. 2003; 425(6958): 612-614, doi: 10.1038/nature01971, indexed in Pubmed: 14534585.

15. Xuan F, Hu K, Zhu T, et al. Immunohistochemical evidence of cone-based ultraviolet vision in divergent bat species and implications for its evolution. Comp Biochem Physiol B Biochem Mol Biol. 2012; 161(4): 398-403, doi: 10.1016/j. cbpb.2012.01.005, indexed in Pubmed: 22269122.

16. Zhao H, Xu D, Zhou Y, et al. Evolution of opsin genes reveals a functional role of vision in the echolocating little brown bat (Myotis lucifugus). Biochem Syst Ecol. 2009; 37(3): 154-161, doi: 10.1016/j.bse.2009.03.001.

17. Jeong MJ, Kim HG, Jeon CJ. The organization of melanopsin-immunoreactive cells in microbat retina. PLoS One. 2018; 13(1): e0190435, doi: 10.1371/journal.pone.0190435, indexed in Pubmed: 29304147.

18. Park EB, Jeon JY, Jeon CJ. Identification of protein kinase $\mathrm{C} \alpha$ - and tyrosine hydroxylase-immunoreactive cells in the microbat retina. Histol Histopathol. 2018; 33(10): 1059-1073, doi: 10.14670/HH-18-001, indexed in Pubmed: 29741202.

19. Butz E, Peichl L, Müller B. Cone bipolar cells in the retina of the microbat Carollia perspicillata. J Comp Neurol. 2015; 523(6): 963-981, doi: 10.1002/cne.23726, indexed in Pubmed: 25521284.

20. Pettigrew JD, Dreher B, Hopkins CS, et al. Peak density and distribution of ganglion cells in the retinae of microchiropteran bats: implications for visual acuity. Brain Behav Evol. 1988; 32(1): 39-56, doi: 10.1159/000116531, indexed in $\mathrm{Pu}-$ bmed: 3191381

21. Glezer II, Hof PR, Leranth C, et al. Calcium-binding protein-containing neuronal populations in mammalian visual cortex: a comparative study in whales, insectivores, bats, rodents, and primates. Cereb Cortex. 1993; 3(3): 249-272, doi: 10.1093/cercor/3.3.249, indexed in Pubmed: 8324371.

22. Gu YN, Kim HG, Jeon CJ. Localization of nitric oxide synthase-containing neurons in the bat visual cortex and colocalization with calcium-binding proteins. Acta Histochem Cytochem. 2015; 48(4): 125-133, doi: 10.1267/ahc.14066, indexed in Pubmed: 26379314.

23. Kim HG, Gu YN, Lee KP, et al. Immunocytochemical localization of the calcium-binding proteins calbindin D28K, calretinin, and parvalbumin in bat visual cortex. Histol Histopathol. 2016; 31(3): 317-327, doi: 10.14670/HH-11-680, indexed in Pubmed: 26536416.

24. Jeong SJ, Jeon CJ. Localization of choline acetyltransferase and tyrosine hydroxylase immunoreactivities in the superior colliculus of the microbat. Histol Histopathol. 2017; 32(6): 609-626, doi: 10.14670/HH-11-826, indexed in Pubmed: 27681043.

25. Abraham AD, Neve KA, Lattal KM. Dopamine and extinction: a convergence of theory with fear and reward circuitry. Neurobiol Learn Mem. 2014; 108: 65-77, doi: 10.1016/j. nlm.2013.11.007, indexed in Pubmed: 24269353.

26. Chinta SJ, Andersen JK. Dopaminergic neurons. Int J Biochem Cell Biol. 2005; 37(5): 942-946, doi: 10.1016/j.biocel.2004.09.009, indexed in Pubmed: 15743669.

27. Jacob SN, Nienborg H. Monoaminergic neuromodulation of sensory processing. Front Neural Circuits. 2018; 12: 51, doi: 10.3389/fncir.2018.00051, indexed in Pubmed: 30042662.
28. Overton PG, Vautrelle N, Redgrave P. Sensory regulation of dopaminergic cell activity: Phenomenology, circuitry and function. Neuroscience. 2014; 282: 1-12, doi: 10.1016/j.neuroscience.2014.01.023, indexed in Pubmed: 24462607.

29. Schultz W. Multiple dopamine functions at different time courses. Annu Rev Neurosci. 2007; 30: 259-288, doi: 10.1146/ annurev.neuro.28.061604.135722, indexed in Pubmed: 17600522.

30. Wise RA. Dopamine, learning and motivation. Nat Rev Neurosci. 2004; 5(6): 483-494, doi: 10.1038/nrn1406, indexed in Pubmed: 15152198.

31. Berger B, Gaspar P, Verney C. Dopaminergic innervation of the cerebral cortex: unexpected differences between rodents and primates. Trends Neurosci. 1991; 14(1): 21-27, doi: 10.1016/0166-2236(91)90179-x, indexed in Pubmed: 1709528.

32. Kosaka T, Hama K, Nagatsu I. Tyrosine hydroxylase-immunoreactive intrinsic neurons in the rat cerebral cortex. Exp Brain Res. 1987; 68(2): 393-405, doi: 10.1007/BF00248804, indexed in Pubmed: 2891559.

33. Phillipson OT, Kilpatrick IC, Jones MW. Dopaminergic innervation of the primary visual cortex in the rat, and some correlations with human cortex. Brain Res Bull. 1987; 18(5): 621-633, doi: 10.1016/0361-9230(87)90132-8, indexed in Pubmed: 2440529.

34. Wang XH, Levitt P, Jenkins AO, et al. Normal development of tyrosine hydroxylase and serotonin immunoreactive fibers innervating anterior cingulate cortex and visual cortex in rabbits exposed prenatally to cocaine. Brain Research. 1996; 715(1-2): 221-224, doi: 10.1016/0006-8993(96)00012-1, indexed in Pubmed: 8739642.

35. Hof P, Glezer I, Revishchin A, et al. Distribution of dopaminergic fibers and neurons in visual and auditory cortices of the harbor porpoise and pilot whale. Brain Res Bull. 1995; 36(3): 275-284, doi: 10.1016/0361-9230(94)00202-c, indexed in Pubmed: 7697381.

36. Lewis DA, Campbell MJ, Foote SL, et al. The distribution of tyrosine hydroxylase-immunoreactive fibers in primate neocortex is widespread but regionally specific. J Neurosci. 1987; 7(1): 279-290, doi: 10.1523/JNEUROSCI.07-01-00279.1987, indexed in Pubmed: 2879896.

37. Gaspar P, Berger B, Febvret A, et al. Tyrosine hydroxylase-immunoreactive neurons in the human cerebral cortex: a novel catecholaminergic group? Neurosci Lett. 1987; 80(3): 257-262, doi: 10.1016/0304-3940(87)90464-2, indexed in Pubmed: 2891090.

38. Björklund A, Dunnett SB. Dopamine neuron systems in the brain: an update. Trends Neurosci. 2007; 30(5): 194-202, doi: 10.1016/j.tins.2007.03.006, indexed in Pubmed: 17408759.

39. Nieoullon A, Cheramy A, Glowinski J. Release of dopamine evoked by electrical stimulation of the motor and visual areas of the cerebral cortex in both caudate nuclei and in the substantia nigra in the cat. Brain Res. 1978; 145(1): 69-83, doi: 10.1016/0006-8993(78)90797-7, indexed in Pubmed: 638784.

40. Törk I, Turner S. Histochemical evidence for a catecholaminergic (presumably dopaminergic) projection from the ventral mesencephalic tegmentum to visual cortex in the cat. Neurosci Lett. 1981; 24(3): 215-219, doi: 10.1016/0304-3940(81)901592, indexed in Pubmed: 6168976.

41. Piraccini, R. Rhinolophus ferrumequinum. The IUCN Red List of Threatened Species. 2016. Available at: https://dx.doi. org/10.2305/IUCN.UK.2016-2.RLTS.T19517A21973253.en. Accessed June 8, 2018.

42. Jeon YK, Kim TJ, Lee JY, et al. AII amacrine cells in the inner nuclear layer of bat retina: identification by parvalbumin immunoreactivity. Neuroreport. 2007; 18(11): 1095-1099, doi: 10.1097/ WNR.0b013e3281e72afe, indexed in Pubmed: 17589306. 
43. Kim GH, Kim HG, Jeon CJ. Immunocytochemical localization of choline acetyltransferase in the microbat visual cortex. Acta Histochem Cytochem. 2018; 51(5): 153-165, doi: 10.1267/ahc.18018, indexed in Pubmed: 30510329.

44. Park EB, Gu YN, Jeon CJ. Immunocytochemical localization of cholinergic amacrine cells in the bat retina. Acta Histochem. 2017; 119(4): 428-437, doi: 10.1016/j.acthis.2017.04.009, indexed in Pubmed: 28483062.

45. Jang YJ, Yu SH, Lee ES, et al. Two types of tyrosine hydroxylase-immunoreactive neurons in the zebrafish retina. Neurosci Res. 2011; 71(2): 124-133, doi: 10.1016/j.neures.2011.07.002, indexed in Pubmed: 21784111.

46. Kruger JL, Dell LA, Bhagwandin A, et al. Nuclear organization of cholinergic, putative catecholaminergic and serotonergic systems in the brains of five microchiropteran species. J Chem Neuroanat. 2010; 40(3): 210-222, doi: 10.1016/j. jchemneu.2010.05.007, indexed in Pubmed: 20566329.

47. Dell LA, Kruger JL, Bhagwandin A, et al. Nuclear organization of cholinergic, putative catecholaminergic and serotonergic systems in the brains of two megachiropteran species. J Chem Neuroanat. 2010; 40(2): 177-195, doi: 10.1016/j. jchemneu.2010.05.008, indexed in Pubmed: 20566331.

48. Maseko BC, Manger PR. Distribution and morphology of cholinergic, catecholaminergic and serotonergic neurons in the brain of Schreiber's long-fingered bat, Miniopterus schreibersii. J Chem Neuroanat. 2007; 34(3-4): 80-94, doi: 10.1016/j. jchemneu.2007.05.004, indexed in Pubmed: 17560075.

49. Jeon CJ, Spencer R, Mize R. Organization and synaptic connections of cholinergic fibers in the cat superior colliculus. J Comp Neurol. 1993; 333(3): 360-374, doi: 10.1002/ cne.903330305, indexed in Pubmed: 8349848.

50. Raghanti MA, Spocter MA, Stimpson CD, et al. Species-specific distributions of tyrosine hydroxylase-immunoreactive neurons in the prefrontal cortex of anthropoid primates. Neuroscience. 2009; 158(4): 1551-1559, doi: 10.1016/j.neuroscience.2008.10.058, indexed in Pubmed: 19041377.

51. Grobin AC, Deutch AY. Dopaminergic regulation of extracellular gamma-aminobutyric acid levels in the prefrontal cortex of the rat. J. Pharmacol. Exp. Ther. 1998; 285(1): 350-357, indexed in Pubmed: 9536031.

52. Medeiros HH, Santana MA, Leite MD, et al. The cytoarchitectonic and TH-immunohistochemical characterization of the dopamine cell groups in the substantia nigra, ventral tegmental area and retrorubral field in a bat (Artibeus planirostris). Neurosci Res. 2016; 112: 37-46, doi: 10.1016/j.neures.2016.06.005, indexed in Pubmed: 27349153.

53. Calvey T, Patzke N, Kaswera-Kyamakya C, et al. Organization of cholinergic, catecholaminergic, serotonergic and orexinergic nuclei in three strepsirrhine primates: Galago demidoff, Perodicticus potto and Lemur catta. J Chem Neuroanat. 2015; 70: 42-57, doi: 10.1016/j.jchemneu.2015.10.002, indexed in Pubmed: 26562782.

54. Shuler MG, Bear MF. Reward timing in the primary visual cortex. Science. 2006; 311(5767): 1606-1609, doi: 10.1126/ science.1123513, indexed in Pubmed: 16543459.

55. Tan CO. Anticipatory changes in regional cerebral hemodynamics: a new role for dopamine? J Neurophysiol. 2009; 101(6): 2738-2740, doi: 10.1152/jn.00141.2009, indexed in Pubmed: 19321643.

56. Zaldivar D, Goense J, Lowe SC, et al. Dopamine is signaled by mid-frequency oscillations and boosts output layers visual information in visual cortex. Curr Biol. 2018; 28(2): 224-235.e5, doi: 10.1016/j.cub.2017.12.006, indexed in Pubmed: 29307559.

57. Tressler J, Schwartz C, Wellman P, et al. Regulation of bat echolocation pulse acoustics by striatal dopamine. J Exp Biol. 2011; 214(Pt 19): 3238-3247, doi: 10.1242/jeb.058149, indexed in Pubmed: 21900471.

Submitted: 20 January, 2020

Accepted after reviews: 9 May, 2020 Available as AoP: 2 June, 2020 